\title{
Experimental study of a passive inverted pendulum control system
}

\author{
Diogo V. Resende ${ }^{1, *}$; Marcus V. G. de Morais ${ }^{1,2}$; Suzana M. Avila ${ }^{1}$ \\ ${ }^{1} \mathrm{PPG}$, Graduate program in Engineering Material Integrity, University of Brasilia (UnB), Campus of \\ Gama (FGA), 72444-240, Gama (Brasilia), Brazil. \\ ${ }^{2} \mathrm{PPG}$, Graduate program in Mechanical Science, University of Brasilia (UnB), Campus Darcy Ribeiro, \\ 70910-900, Brasilia, Brazil.
}

\begin{abstract}
This work presents the results obtained by an experimental study of vibration control for a system with a single degree of freedom, composed by a sliding base on an air track. An inverted pendulum-type tuned mass damper (TMD-IP) is installed on the sliding base to reduce its displacements. First, a reduced model is built using a 3D printer. The experimental model consists of two parts: the mains system, which is the sliding base; and the inverted pendulum (TMD-IP). The model can be tested as a one-degree-of-freedom system using only the base or as a two-degree system of freedom system after connecting the TMD-IP to the sliding base. The CIDEPE air rail is used to provide the sliding base displacement, which will receive the TMD-IP. The acquisition of the response data in the time domain is done through the software CvMob, which performs image acquisition and was produced by the Nucleus of Technological Innovation in Rehabilitation - NITRE, Federal University of Bahia.
\end{abstract}

\section{Introduction}

There are several ways to avoid the effects of excessive vibration, among them passive structural control. Structural control promotes changes of the stiffness and damping properties of a given structure. For example by installing devices in which some of the energy that a dynamic load applies to the main structure is absorbed or transferred. An example of this type of control is inverted pendulum systems functioning as tuned mass dampers, TMDIP [1].

Anh et al. [2] compared the efficiency of the control exerted by an inverted pendulum and a simple pendulum on an inverted pendulum-type main system and found the best performance for the TMD-IP. Majcher\&Wójcicki [3] analysed numerically, not only the influence of vertical oscillations caused by an earthquake in a tall building, but also the efficiency of a simple pendulum type tuned mass damper (TMD), as a control system.

Colherinhas et al. [4] analysed numerically the dynamic behaviour of a slender tower before and after the installation of a simple pendulum-type TMD and an optimization was performed with genetic algorithm to improve the efficiency of the control system.

Guimarães and Avila [5] studied the dynamic stability of a wind turbine modelled as an inverted pendulum. Deraemaeker\&Soltani [6] started a study with the intention of obtaining, in an analytical way, the solution for the optimization of a simple pendulum type TMD, connected to a main system considered without damping and reduced to a model with one degree of freedom.

In this work, a TMD-IP type is designed and built, as a reduced model, and its efficiency is tested on an experimental bench built on an air rail. The first case analysed is the main system with one degree of freedom (DOF) uncontrolled. Subsequently, the TMD-IP is

${ }^{*}$ Corresponding author: diogocivil@gmail.com 
connected to the sliding base. This damper, initially, consists only of an inflexible rod. Then, a small tip mass is connected and new tests are performed. Dynamic system responses are recorded through an image acquisition system and then analysed. The results obtained with the use of the proposed control system were satisfactory.

\section{Theoretical model and mathematical formulation}

In this work, a tuned mass damper (TMD) with inverted pendulum geometry is coupled to a system with translational degree of freedom, as shown in Figure 1 (a). Figure 1 (b) shows the corresponding experimental model of the system. The main system has one degree of freedom and can translate horizontally. The mass of this system $m_{c}$, is connected to dampers with damping constant $c / 2$ and springs with elastic constant $k_{c} / 2$. The inverted pendulum TMD (TMD-IP) control system consists of an inflexible rod connected to the main system by means of a rotational spring $k$. This system has two degrees of freedom and the rod, with linear density $\rho$ and length $l$, has a degree of rotational freedom around its connection at the base. The TMD-IP system may have a mass $m$ connected to the upper end of the rod.

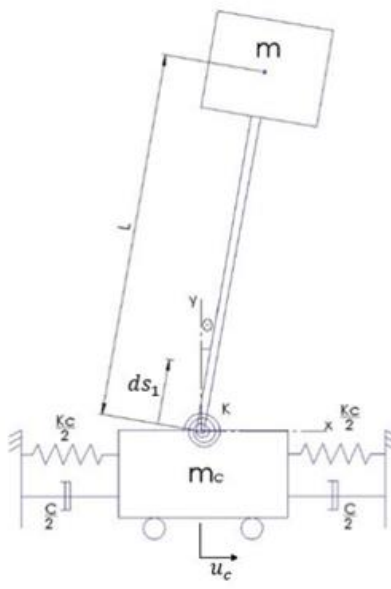

(a)

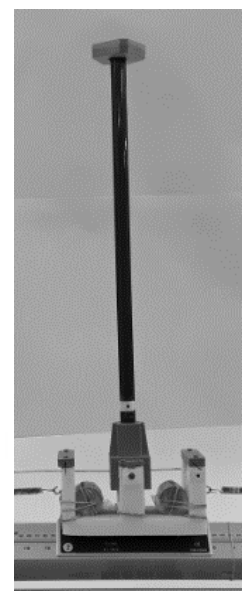

(b)

Fig. 1. (a) Theoretical model [5]; (b) Experimental model.

Equation (1) represents the matrix motion equation of the system with the displacement vector $\boldsymbol{u}_{\boldsymbol{c}}$, velocities vector $\dot{\boldsymbol{u}}_{\boldsymbol{c}}$, accelerations vector $\ddot{\boldsymbol{u}}_{\boldsymbol{c}}$, loading vector $\boldsymbol{f}(t)$, in addition to mass, damping and stiffness matrices, which are represented by $\boldsymbol{M}, \boldsymbol{C}$ and $\mathbf{K}$, respectively. In Equation (2), the matrices and vectors of the motion equation are detailed.

$$
\begin{gathered}
\boldsymbol{M} \ddot{\boldsymbol{u}}_{\boldsymbol{c}}+\boldsymbol{C} \dot{\boldsymbol{u}}_{\boldsymbol{c}}+\boldsymbol{K} \boldsymbol{u}_{\boldsymbol{c}}=\boldsymbol{f}(t) \\
{\left[\begin{array}{cc}
\frac{\rho \cdot l^{3}}{3}+m \cdot l^{2} & m \cdot l+\frac{\rho \cdot l^{2}}{2} \\
m \cdot l+\frac{\rho \cdot l^{2}}{2} & m_{c}+m+\rho \cdot l
\end{array}\right]\left[\begin{array}{c}
\ddot{\theta} \\
\ddot{u}_{c}
\end{array}\right]+\left[\begin{array}{ll}
0 & 0 \\
0 & c
\end{array}\right]\left[\begin{array}{c}
\dot{\theta} \\
\dot{u}_{c}
\end{array}\right]} \\
+\left[\begin{array}{cc}
k-m \cdot g \cdot l-\frac{\rho \cdot g \cdot l^{2}}{2} & 0 \\
0 & K_{c}
\end{array}\right]\left[\begin{array}{c}
\theta \\
u_{c}
\end{array}\right]=\left[\begin{array}{c}
0 \\
f(t)
\end{array}\right]
\end{gathered}
$$


where $g, f(t), \theta, u_{c}$, represent: acceleration of gravity, applied excitation, rotational displacement and translational displacement.

\section{Experimental methodology}

To verify the effectiveness of the adopted passive control system, a reduced experimental model is developed with the aid of a 3D printer. The model consists of two main parts, the base and the inverted pendulum. The first part represents a system with translational 1DOF. The inverted pendulum can be connected to the base, thus constituting a model representing a 2DOF system (Figure 1 (b)). In order to enable the horizontal translation movement, the CIDEPE air rail, shown in Figure 2, is used. An initial displacement of 2,5 $\mathrm{cm}$ is applied as excitation in all cases analysed and the tests were repeated five times.

Data acquisition in time domain is performed through image acquisition techniques. For this, a cell phone device Samsung Galaxy J5 Prime (capture speed of 30fps and photo definition $720 \times 1280$ ) and the software CvMob is used. Data analysis and processing are performed in MATLAB software, with the aid of the curve fitting tool - CFTOOL. The damped natural frequencies, obtained by the mentioned procedure, are compared with those obtained by means of the Fourier fast transform - FFT.

The geometric and mass parameters of the experimental model are: $m_{c}=1133,17 \mathrm{~g}$, $m=37 \mathrm{~g}, \rho=0,29 \mathrm{~kg} / \mathrm{m}, l=0,52 \mathrm{~m}$ and $g=9,78 \mathrm{~m} / \mathrm{s}^{2}$.

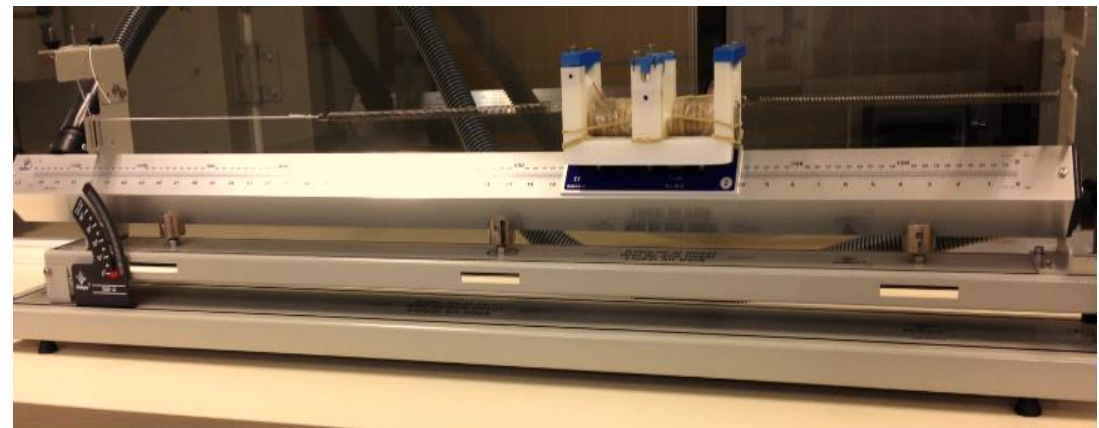

Fig. 2. 3D model with 1 DOF on the air rail.

\subsection{Signal identification in time domain}

After the acquisition of the system response data in time domain, from the capture of the images, the information is imported into MATLAB in order to perform the data processing with CFTOOL. The equation of the curve used for the adjustment of the points is shown in Equation (3), being composed of two damped sinusoids, causing the adjusted response of the system to contemplate the influence of its two modes of vibration. Parameters $b$ and $c$ are determined by curve fitting and, using Equation (4), undamped natural frequencies and damping ratio are calculated.

$$
\begin{gathered}
u_{c}(t)=A_{1} e^{-b_{1} t} \sin \left(c_{1} t+\phi_{1}\right)+A_{2} e^{-b_{2} t} \sin \left(c_{2} t+\phi_{2}\right)+e \\
b_{i}=\xi \omega_{n} \quad, \quad c_{i}=\omega_{d} \quad, \quad \omega_{n}=\sqrt{c^{2}+b^{2}} \quad, \quad \xi=\frac{b}{\omega_{n}} \quad e \quad(i=1,2)
\end{gathered}
$$

The values of the damped natural frequencies $\left(\omega_{d}\right)$ found with the procedure described above are compared with the frequencies found in the frequency response function obtained by applying the Fourier fast transform (FFT) in the time signal. 


\subsection{Results}

The first part of the analysis contemplates the characterization of the stiffness constants $k_{c}$ and $k$, which have a direct influence on the frequency and damping values of the systems that will be analysed later.

The translational stiffness coefficient $k_{c}$, connected to the base, is obtained from the static loading test, by measuring the deformation in the elastic element after each incremental increase in mass. A linear fit is made to the obtained results and the tangent of that line is the parameter, $k_{c} / 2=164,71 \mathrm{~N} / \mathrm{m}$, sought. This value corresponds to the stiffness of each of the two springs that are connected in parallel. The Pearson correlation coefficient for the analysis is $R_{p}=0.9999$.

The coefficient of rotational stiffness $k$, of the connection between the inverted pendulum and the base, is obtained from the equation that relates the natural frequency of the pendulum with the parameters of the system, according to [7]. The relation quoted is represented in Equation (5).

A free-vibration test for the pendulum alone was performed to determine its undamped natural frequency, which is an input parameter for the equation quoted. In this test only, the pendulum has freedom of movement (rotation), while the base is prevented from translating, that is, the base is fixed in the air rail. The values found for this test were: $\omega_{d}=15,52 \pm 0,09[\mathrm{rad} / \mathrm{s}], \quad \omega_{n}=15,52 \pm 0,09[\mathrm{rad} / \mathrm{s}] \quad$ e $\quad \xi=0,99 \pm 0,17 \% \quad$ (using CFTOOL); and $\omega_{d}=15,46 \pm 0,00[\mathrm{rad} / \mathrm{s}]$ (using FFT).

$$
\omega_{n, \theta}=\sqrt{\frac{6 k-g l(6 m+3 \rho l)}{2 l^{2}(\rho l+3 m)}}
$$

The calculated stiffness parameter is $k=1,45 \mathrm{Nm} / \mathrm{rad}$. The frequency values presented in Table 1 are quite close, but they have small differences in the decimal places. The confidence interval used considers $95.5 \%$ of the values $(2 \sigma)$ and the damping ratio found indicates a very low damping level.

Table 1 shows the damped natural frequencies $\left(\omega_{d}\right)$ and the damping ratios $(\xi)$ of the systems analysed with and without control, obtained through the two different techniques. For the three experimental cases, the confidence interval used in the evaluation of the experimental error contemplates $95,5 \%$ of the values $(2 \sigma)$.

Figure 2 represents the experimental model with 1 DOF on the air rail. The frequency values are quite close, but they have small differences in the second decimal place. The damping ratio found indicates a very low damping level.

The experimental model with 2DOF analysed following is similar to the model shown in Figure 1 (b), however, a mass is not coupled to the upper end of the pendulum. The frequency values are quite close when the two techniques are compared. When the values of the natural frequencies of vibration are compared, $\omega_{d 1}$ with $\omega_{d 2}$, we can see that they are also close. The damping ratios found indicate a very low level of damping.

Next, the experimental model shown in Figure 1(b) is tested. The TMD-IP is composed of a mass connected to the upper end of the inflexible rod. Frequency values are quite close when the two techniques used for identification are compared. The damping ratios found for the second vibration mode indicate a very low damping level as well.

The frequencies found for the first vibration mode, $\omega_{d 1}=0$, indicate a condition of possible global divergent instability for the system. From the analysis of the element of the stiffness matrix presented in Equation (2), associated to the degree of freedom $\theta$, it is possible to determine the quantity of mass $m$, from which the system becomes unstable and subject 
to tipping. By making the term $k_{1,1}$, of the stiffness matrix, positive, the limiting mass ( $m \leq$ $210 \mathrm{~g}$ ) is determined for the condition of divergent instability.

Table1. Damped natural frequencies $\omega_{d}$ damping ratios $\xi$.

\begin{tabular}{|c|c|c|c|c|c|c|}
\hline & \multicolumn{4}{|c|}{ CFTOOL } & \multicolumn{2}{c|}{ FFT } \\
\cline { 2 - 7 } & $\omega_{d 1}[\mathrm{rad} / \mathrm{s}]$ & $\omega_{d 2}[\mathrm{rad} / \mathrm{s}]$ & $\xi_{1}[\%]$ & $\xi_{2}[\%]$ & $\omega_{d 1}[\mathrm{rad} / \mathrm{s}]$ & $\omega_{d 2}[\mathrm{rad} / \mathrm{s}]$ \\
\hline 1DOF & $\begin{array}{c}16,77 \pm \\
0,00\end{array}$ & $* * *$ & $\begin{array}{c}0,14 \pm \\
0,01\end{array}$ & $* * *$ & $\begin{array}{c}16,75 \pm \\
0,00\end{array}$ & $* * *$ \\
\hline $\begin{array}{c}\text { 2DOF } \\
\text { Pendulum }\end{array}$ & $\begin{array}{c}14,22 \pm \\
0,01\end{array}$ & $\begin{array}{c}17,38 \pm \\
0,02\end{array}$ & $\begin{array}{c}0,61 \pm \\
0,05\end{array}$ & $\begin{array}{c}0,45 \pm \\
0,06\end{array}$ & $\begin{array}{c}14,28 \pm \\
0,20\end{array}$ & $\begin{array}{c}17,49 \pm \\
0,00\end{array}$ \\
\hline $\begin{array}{c}\text { 2DOF } \\
\text { Pendulum } \\
\text { +Mass }\end{array}$ & $\begin{array}{c}0,00 \pm \\
0,00\end{array}$ & $\begin{array}{c}16,28 \pm \\
0,05\end{array}$ & $\begin{array}{c}99,64 \pm \\
1,60\end{array}$ & $\begin{array}{c}0,19 \pm \\
0,01\end{array}$ & $\begin{array}{c}0,00 \pm \\
0,00\end{array}$ & $\begin{array}{c}16,25 \pm \\
0,17\end{array}$ \\
\hline
\end{tabular}

* Frequency values equal to 0.00 have a magnitude smaller than the adopted precision.

Figure 3 presents the time domain responses in terms of sliding base displacement for three distinct cases: (a) sliding base without control; (b) base with TMD-IP composed only by the inflexible rod; and (c) TMD-IP base composed of a mass connected to the upper end of the inflexible rod. It can be concluded that the most efficient system was the TMD-IP without the tip mass. This system was the one that most diminished the maximum initial amplitudes of the sliding base and that most reduced the time spent for the displacement reduction.

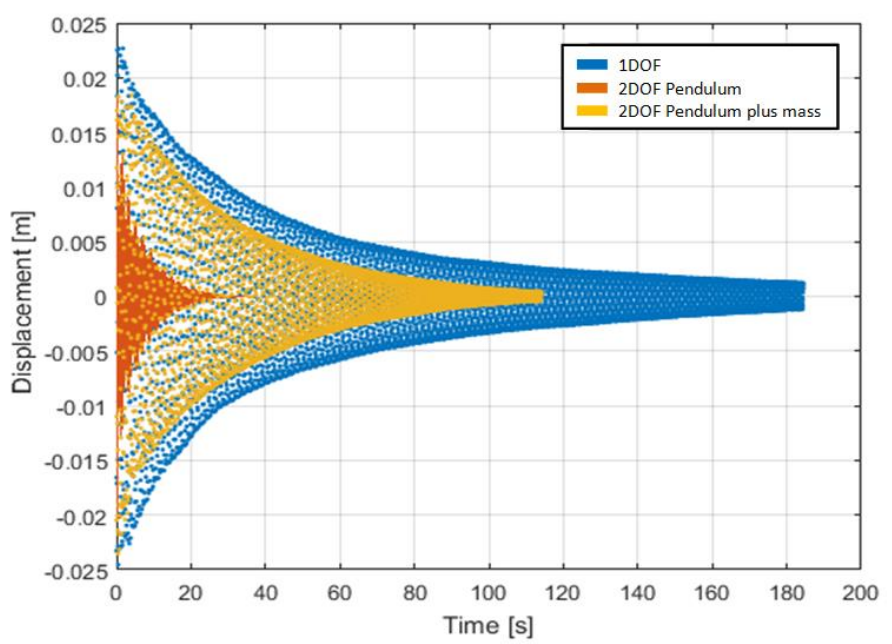

Fig. 3. Sliding base displacements with and without control.

\section{Conclusions}

The methodology used for the acquisition of the images was satisfactory, images and signals obtained presented good quality. The comparison between the damped natural frequencies $\left(\omega_{d}\right)$ obtained using the CFTOOL and FFT tools showed very small differences, generally in the second decimal place, which can be considered insignificant. In addition, tests presented good repeatability, since the associated errors are also small, being restricted to the decimal places.

The values obtained for the damping ratios $(\xi)$, lower than $1 \%$, indicate very low damped systems and, depending on the analysis, it is possible to consider the system undamped. 
All analysed systems had low frequencies, always lower than $3 \mathrm{~Hz}$. The system with the TMD-IP installed, without the tip mass, presented the best performance. It reduced the maximum initial amplitude of the system with 1 DOF approximately $45 \%$; and reduced its damping time by approximately $83 \%$.

The mass of $37 \mathrm{~g}$, connected to the end of the AMS-PI is lower from the mass of $210 \mathrm{~g}$, calculated for the instability limit, however, when this mass was installed at the end of the TMD-IP, the control system lost efficiency.

The value of $k_{c}$ obtained experimentally has a high coefficient of correlation of Pearson $(R=0.9999)$, which indicates a high correlation between the experimental points and the adjusted line.

As a future perspective for the improvement of this research, it is intended to attach a device with frequency adjustment to excite the systems harmonically, which were analysed in this work only with initial displacement applied.

This work was supported by the Coordination for the Improvement of Higher Education Personnel (CAPES) and the Federal District Foundation for Research Support (FAPDF).

\section{References}

[1] F.S. Oliveira, A.L. Zuluaga, S.M. Avila, J.L.V. Brito, Int. J. of Innovations in Mat. Science and Eng.1, 82-89 (2014).

[2] N.D. Anh, H. Matsuhisa, L.D. Vieta, L. D., M. Yasuda, J. of Sound and Vib., 307, 187201 (2007) 187-201.

[3] K. Majchern, Z. Wojcicki, Z., Arch. of Civil and Mech. Eng.,14, 204-217(2014).

[4] G.B. Colherinhas, M.V.G. Morais, M.A.M. Shzu, S.M. Avila, Proc. of COMPDYN (2015).

[5] P. V. B. Guimarães, M. V. G. Morais, S.M. Avila, Vibration Engineering and Technology of Machinery, Springer International Publishing Switzerland Mechanisms and Machine Science 23, 379-388 (2014).

[6] A. Deraemaeker, P. Soltani, J Multi-body Dynamics, 231, 285-291(2017).

[7] P.L. Bernardes, S.M. Avila, S. M., G.B. Colherinhas, M.V.G. Morais, Procc. of XXXVIII Ibero-Latin American Cong. on Comp. Met. in Eng., CILAMCE, Florianópolis, (2017). 